\title{
Texture based feature extraction methods for content based medical image retrieval systems
}

\author{
Burhan Ergen ${ }^{\mathrm{a}, *}$ and Muhammet Baykara ${ }^{\mathrm{b}}$ \\ ${ }^{a}$ Department of Computer Engineering, Faculty of Engineering, Firat University, 23119, Elazig, \\ Turkey \\ ${ }^{b}$ Department of Software Engineering, Faculty of Technology, Firat University, 23119, Elazig, Turkey
}

\begin{abstract}
The developments of content based image retrieval (CBIR) systems used for image archiving are continued and one of the important research topics. Although some studies have been presented general image achieving, proposed CBIR systems for archiving of medical images are not very efficient. In presented study, it is examined the retrieval efficiency rate of spatial methods used for feature extraction for medical image retrieval systems. The investigated algorithms in this study depend on gray level co-occurrence matrix (GLCM), gray level run length matrix (GLRLM), and Gabor wavelet accepted as spatial methods. In the experiments, the database is built including hundreds of medical images such as brain, lung, sinus, and bone. The results obtained in this study shows that queries based on statistics obtained from GLCM are satisfied. However, it is observed that Gabor Wavelet has been the most effective and accurate method.
\end{abstract}

Keywords: Content-based image retrieval, GLCM, GLRLM, Gabor wavelet

\section{Introduction}

In recent years, the medical images have been used for diagnosis, teaching, and management. One of the required processes in a health care provider is to archive medical images produced by medical imaging devices. The purpose of medical image archives (MIA) is to save the diagnostic medical images of patients on computer environment, make queries on these images, retrieve when they are needed and serve them as the user desires [1,2]. The MIA require an efficient indexing to enable fast access to images in the databases because there are large amounts of medical image to store [3]. Currently used image archiving systems in medical is the PACS that provide access to the images using their metadata information. This information is saved with the image using the DICOM standard. The metadata information is generally the data such as the date of image, patient information and the file number information specified by an operator. In this data, there is no information related with image itself [4].

\footnotetext{
${ }^{*}$ Corresponding author: Burhan Ergen, Department of Computer Engineering, Faculty of Engineering, Firat University 23119, Elazig, Turkey. Tel.: +90 424 2370000/6316; Fax: +90 424 2415526; E-mail: ergen@firat.edu.tr.
}

0959-2989/14/\$27.50 @ 2014 - IOS Press and the authors. 
In order to access the required image and related data and images quickly, variety of techniques has been used and new techniques are researched. In recent years, the methods of the query depending on content data of images have been developed rather than the text-based query using the name of data. In content based image retrieval (CBIR) systems, some features extracted from the images in a database are used for query. In feature extraction, besides using the methods of extracting the statistical information of spatial or frequency domain, the analysis of the image according to color and shape descriptors is possible [5].

The textural features are very important to determine the content of a medical image. Textural features provide scenic depth, the spatial distribution of tonal variation, and surface orientation. Various feature extraction methods such as discrete wavelet frame, HU moment invariant, Fourier transform, gray-level histogram (GLH) and gray-level coherence vector were implemented to build a CBIR system. Some of the most well-known methods are based gray level co-occurrence matrix and gray level run length matrix $[2,6]$. We have examined these methods for extracting features of medical images because these methods achieve some success in classification of textural images [7]. Recently, the human visual system can be modeled as a filter bank represented using Gabor functions having different orientation and frequencies. The output of the representation using Gabor function can be accepted as the responses of the human visual system. In particular, the Gabor wavelet transform has demonstrated good performance in texture representation and discrimination, and has been successfully applied to face recognition, object detection, palm print recognition, and also object tracking $[8,9]$. Therefore, we have experimented Gabor wavelet based feature extraction method to determine its performance for CBIR systems by comparing well known spatial methods.

\section{Methods}

The CBIR use visual identifiers in order to define the images. Those identifiers can be divided into three groups as color, texture and shape. In this study, the images were analyzed for texture descriptors. Statistical approaches include the methods of a spatial area, signal processing methods and modelbased methods. In the methods of spatial features, especially in the second order statistics are obtained because people are more sensitive to the second grade statistics. It is accepted that gray level cooccurrence matrix and gray level variance matrix provides these statistics. The higher-level statistics such as the length of the gray level range, power spectrum and autocorrelation function can be measured. In this study, statistical spatial approach methods have been studied.

The features in spatial domain methods are obtained as a result of some statics. Human visual system is sensitive to this statistical information. The examples of this type of statistics are gray level co-occurrence matrix, the length of the gray level range of matrix, and recently Gabor wavelet transform. The methods of spatial providing general information on probabilistic calculation results of the image are different from frequency-based methods and do not execute any action about visual system and the sub-band images.

\subsection{Gray level co-occurrence matrix}

Gray-level co-occurrence matrix (GLCM) proposed by M. Haralick is a measure of the description of texture's gray-level dependence in spatial domain. For an image having $N_{x}$ columns and $N_{y}$ rows, suppose that gray level at each pixel is quantized to $N g$ levels. If $L_{x}=\left\{1,2, \ldots, N_{x}\right\} L_{y}=\left\{1,2, \ldots, N_{y}\right\}$, 
and $G_{x}=\left\{0,1, \ldots, N_{g}-1\right\}$ are the columns, the rows and the set of $N_{g}$ quantized gray level respectively, the set $L_{x} \times L_{y}$ is the set of pixels of the image ordered by their row-column designations. The image $I$ can be presented as a function that assigns some gray level in $G$ to each pixel in $G$ to each pixel or pair of coordinates in $L_{x} \times L_{y}$. The matrix of relative frequencies $P_{i j}$ with two neighboring pixels specifies the texture -context information [10].

After obtaining the GLCM matrix of the image to be evaluated, some statistical calculations are performed on this matrix to constitute feature vector for comparison. Although many statistical functions has been proposed to use as a feature in feature vector, the most widely accepted statistical methods are energy, entropy, homogeneity, contrast and correlation [7,11]. Hence, we used these five textural features in the constitution of feature vector.

\subsection{Gray level run length matrix}

A large number of neighboring pixels in the same gray level represents a rough texture; few of these pixels describe the thin texture. The lengths of texture run in different directions work as texture definition. A texture's run is maximum close the sequence of constant gray-level pixels which are in a line. Then these can be identified as expression of gray level, length and direction. Texture defining features can base on the calculation of close probabilities of length and gray level of run in texture. Gray level run length method (GLRLM) is based on the calculation of the number of gray levels of different lengths. The length of a gray level is the sequence of the neighboring image points in a linear having the same gray level value. The length of a gray level is the number of image points in it $[12,13]$. Galloway proposed the use of a run length matrix for texture feature extraction. For an image to be analyzed, a run-length matrix $G(i, j)$ is defined as the number of runs with pixels of gray level $i$ and run length $j$. To represent the given image, various texture features can be derived from this runlength matrix [14]. The most commonly used features created by using this matrix are long run emphasis (LRE), short run emphasis (SRE), gray level uniformity (GLU), run length uniformity (RLU). The combinations of these calculations are used to constitute a feature vector.

\subsection{Gabor wavelet filtering}

Gabor wavelet transformation feature is to have similar characteristics with human visual system especially in terms of representation of the frequency and orientation. It separates many filtered image which have changes in a series of limited frequency and trends in intensity. These features are also considered to be appropriate to distinguish the texture [15]. Conversion can be considered as wavelet transform of which main wavelet is Gabor function. The Gabor wavelet transform of an image can be described as below;

$$
g(x, y)=\sum_{s} \sum_{t} f(x-s, y-t) \psi(s, t)
$$

In spatial area, a Gabor filter can be given as below; 


$$
\psi(x, y)=\exp \left\{-\frac{1}{2}\left[\frac{\tilde{x}}{\sigma_{x}}+\frac{\tilde{y}}{\sigma_{y}}\right]\right\} \cos (2 \pi \tilde{x})
$$

Here, $f \mathrm{f}$ is central frequency. $\sigma_{x}$ and $\sigma_{y}$ are, respectively, the constant of space of $x$ and $y$ 's Gaussian casing. After the combining the converted virtual and real parts, in order to gain the size of converted image, the texture features characterizing the image were removed. This process is applied to both the query image and images in the database.

\section{Evaluation criteria}

After getting the feature vectors, Euclidean distance was used to find the amount of similarity. Euclidean distances are not affected by the addition of new objects that may be unusual for clustering analysis. But, the differences in scale between dimensions significantly affect the Euclidean distances. Euclidean distance formula is the most commonly used formula in calculation.

$$
\operatorname{Distance}(x, y)=\left\{\sum_{i}\left(x_{i}-y_{i}\right)^{2}\right\}^{1 / 2}
$$

Two basic scales to measure our success is used; precision and recall. The evolution of visual access systems having large collection is a hard but necessary process to fulfill like text-based information retrieval system. Precision and recall evaluation criteria applied in traditional information retrieval systems are also used in the evaluation of image access systems. When we search in terms of user subjectivity, the formulas of precision and recall are given as follows [16,17];

$$
\begin{aligned}
& \text { Precision }=\frac{|A(q) \cap R(q)|}{|A(q)|} \\
& \text { Recall }=\frac{|A(q) \cap R(q)|}{|R(q)|}
\end{aligned}
$$

Where R (q) is the data set related to the query, A (q) is the query result. To verbalize, precision in system evaluation is the ratio of the numbers of images obtained as a result of the query to the number of the entire images after the query. Recall is, on the other hand, the ratio of the numbers of images obtained as a result of the query to all of the images on the system. Recall measures the power of system in accessing all the relevant documents. Precision only measures the power of system in finding documents that are required. In this study, we just focused on precision results because the sample database has certain number known images.

\section{Implementation and results}

In this part, the success of algorithms taken from features in our database in which biomedical 
content is available will be analyzed. Methods are tested without a special environment controlling the color, formal specifications, resolution, field of view angle or the lighting. Figure 1 shows sample image from the image database. In implementations, the success of methods to bring images similar to demanded images measured. Approximately 8500 units to be used in biomedical images archived in the database. The image archive consists of images of brain, lung, bone, eye, and sine. Images in the archive are 24-bit gray-level deep. The known methods were tested on the database after creating a database having total 10 sample databases consisting of 100 elements. Different features of gray-level co-occurrence matrix are calculated and according to those results an access analysis was performed.

Table 1 shows the chart of statistical methods used as feature vector. The symbol "+" denotes that feature is included in feature set. In Figure 2, in case of the use of features given in Table 1, and if 10 samples are used for the sample database, the average percentage of true access is given, precision.

Table 1

The chart of features for GLCM

\begin{tabular}{lllllll}
\hline \multirow{2}{*}{ Features } & \multicolumn{7}{l}{ Feature Set Number } \\
\cline { 2 - 7 } & 1 & 2 & 3 & 4 & 5 & 6 \\
\hline Entropy & + & - & - & - & + & - \\
\hline Energy & - & + & - & - & + & + \\
\hline Homogeneity & - & - & + & - & + & + \\
\hline Contrast & - & - & - & + & + & + \\
\hline Correlation & - & - & - & - & + & + \\
\hline
\end{tabular}

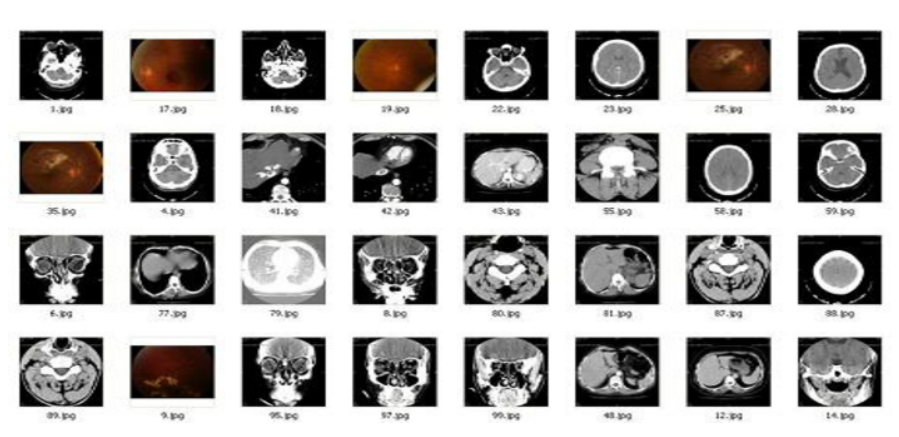

Fig. 1. A Sample taken from created database.

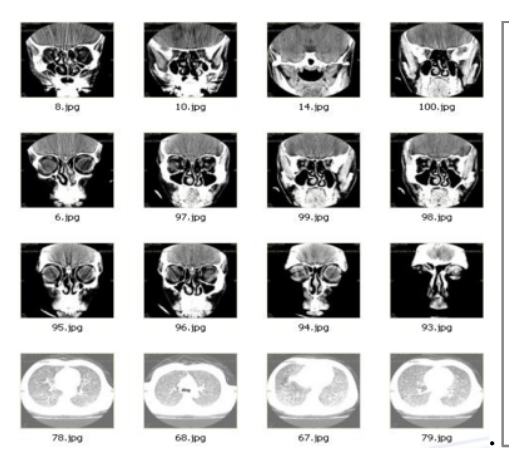

(a)

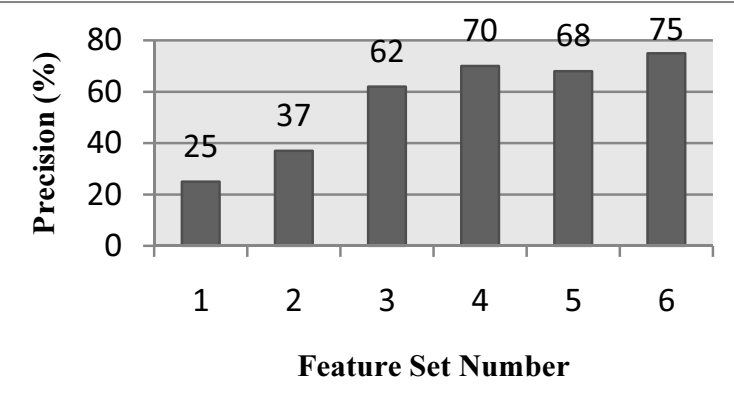

(b)

Fig. 2. GLCM query results: (a) A sample query and (b) Average precision results. 
Table 2 show combinations of statistical methods used feature vector for GLRLM. Figure 3(a) shows a sample query for GLRLM as the first image is the searched image. Figure 3(b) shows the average precision for GLRLM. Figure 4 shows a sample query and the average precision for Gabor wavelet. The most similar image is shown in the first order from left to right, later the images in second order and therefore it continues until the last image. In the experiments, we have observed that space constant and Gabor filter has no significant effect on the access rate of orientation parameter. It was an expected result that the constants and orientation parameter shall not change the adequacy of access.

Table 2

The chart of features used for GLRLM

\begin{tabular}{lllll}
\hline \multirow{2}{*}{ Features } & \multicolumn{4}{l}{ Feature Set Number } \\
\cline { 2 - 5 } & 1 & 2 & 3 & 4 \\
\hline RLU & - & - & - & + \\
\hline SRE & - & - & + & + \\
\hline LRE & - & + & + & + \\
\hline GLU & + & + & + & + \\
\hline
\end{tabular}

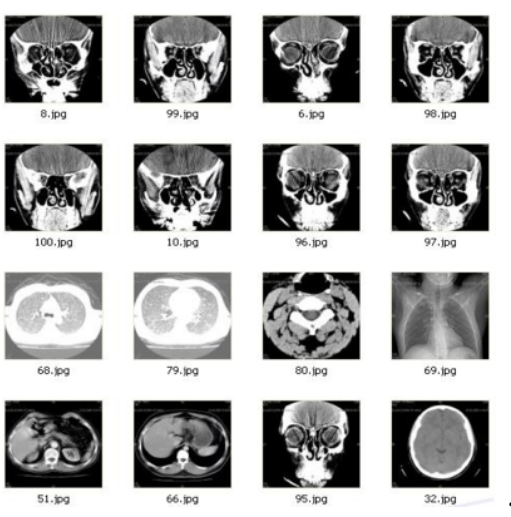

(a)

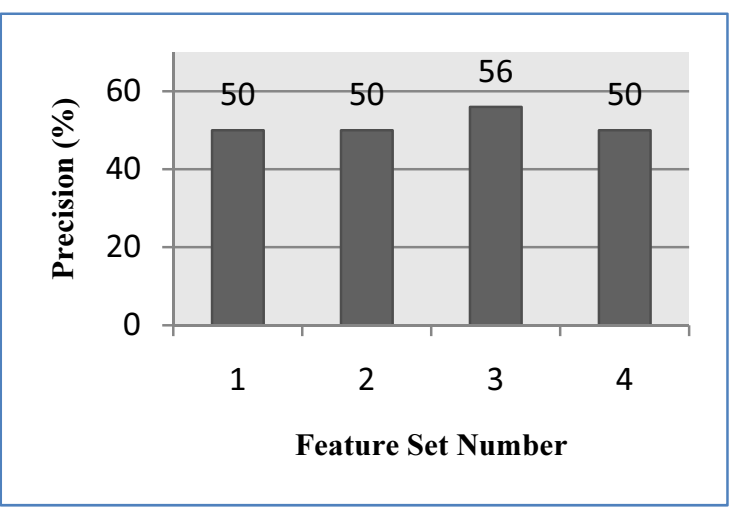

(b)

Fig. 3. GLRLM query results: (a) A sample query and (b) Average precision results.

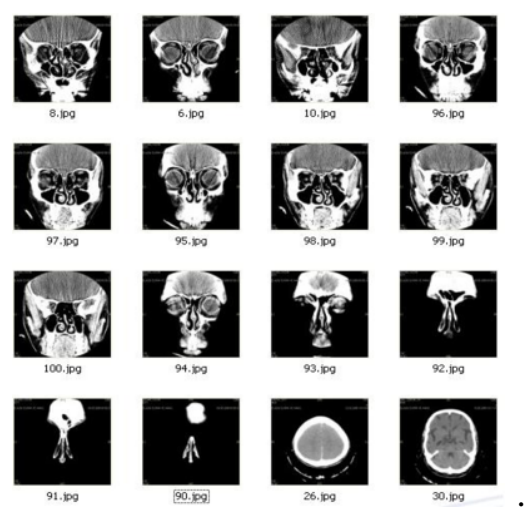

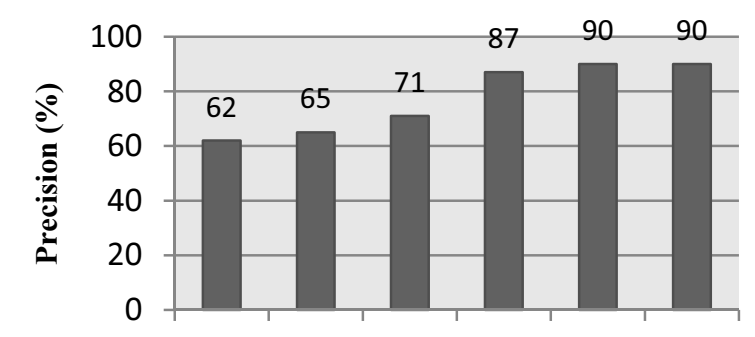

$\pi / 1.2 \pi / 1.3 \pi / 1.4 \pi / 1.5 \pi / 1.6 \pi / 1.7$

Wavelet Frequency (rad)

Fig. 4. Gabor wavelet results: (a) A sample query and (b) precision result. 
Space constants cannot have the ability to destroy the features separating an image from the other. Therefore, since they do not have the knowledge of the direction of images in database $(\theta)$, orientation parameter may not have distinctive features. The images in the database are general-purpose images and do not indicate a pathological condition relevant to a particular region of the human body or texture. If we concerned with finding a particular image, similar to others in the database where face or fingerprint images are available, orientation factor, would give distinctive features. Therefore, these parameters, respectively, $\sigma_{x}=\pi / 2, \sigma_{y}=\pi / 2$ and $\theta=2 \pi / 3$ are selected as constant dominant values. Frequency parameter is observed to be quite distinctive.

\section{Conclusion}

Content-based image retrieval has become an important area in data visualization and database creation. In this study performed on medical images, it is observed that Gabor Wavelet has been the most effective method. These sixteen-images are found by access method described above and are the most similar to the query image. It is obvious at Figure 3(a) that the first images are very similar to the given images; but the last images are not related with the desired image. Figure 4(b) shows the performance of Gabor wavelet transform for different frequencies. The average access rate is obtained by ten times test procedure. For this reason, in order to measure the adequacy of the average accesses, applying to recall definition, ten sub- sequences that consist of a hundred images which we know well in database are created. The results indicate that in order to find the textural features of the image, wavelength of Gabor function is the most critical factor. If the frequency increases, the average access rate will be better for a while.

\section{References}

[1] T.M. Lehmann, M.O. Güld, T. Deselaers, D. Keysers, H. Schubert, K. Spitzer, H. Ney and B.B. Wein, Automatic categorization of medical images for content-based retrieval and data mining, Computerized Medical Imaging and Graphics 29 (2005), 143-155.

[2] H. Müller, N. Michoux, D. Bandon and A. Geissbuhler, A review of content-based image retrieval systems in medical applications - clinical benefits and future directions, International Journal of Medical Informatics 73 (2004), 1-23.

[3] A. Kak and C. Pavlopoulou, Content-based image retrieval from large medical databases, in: 2002 Proceedings of First International Symposium on 3D Data Processing Visualization and Transmission, IEEE, 2002, pp. 138-147.

[4] M.Y. Law, H. Huang, X. Zhang and J. Zhang, The data model of a PACS-based DICOM radiation therapy server, in: Medical Imaging, International Society for Optics and Photonics, 2003, pp. 118-129.

[5] S. Atnafu, R. Chbeir and L. Brunie, Content-based and metadata retrieval in medical image database, in: Proceedings of the 15th IEEE Symposium on Computer-Based Medical Systems (CBMS 2002), 2002, pp. 327-332.

[6] K. Seetharaman and M. Kamarasan, Statistical framework for image retrieval based on multiresolution features and similarity method, Multimedia Tools and Applications 1 (2013), 1-20.

[7] A. Baraldi and F. Parmiggiani, An investigation of the textural characteristics associated with gray level cooccurrence matrix statistical parameters, IEEE Transactions on Geoscience and Remote Sensing 33 (1995), 293-304.

[8] L. Shen and L. Bai, A review on Gabor wavelets for face recognition, Pattern Analysis \& Applications 9 (2006), 273292.

[9] H. Palaio and J. Batista, Kernel based multi-object tracking using gabor functions embedded in a region covariance matrix, Pattern Recognition and Image Analysis 1 (2009), 72-79.

[10] L.-K. Soh and C. Tsatsoulis, Texture analysis of SAR sea ice imagery using gray level co-occurrence matrices, IEEE Transactions on Geoscience and Remote Sensing 37 (1999), 780-795.

[11] S. Selvarajah and S. Kodituwakku, Analysis and comparison of texture features for content based image retrieval, International Journal of Latest Trends in Computing 2 (2011), 108-113. 
[12] A. Chu, C. Sehgal and J.F. Greenleaf, Use of gray value distribution of run lengths for texture analysis, Pattern Recognition Letters 11 (1990), 415-419.

[13] D. Marhefka, N. Venkataraman, A. Khan, S. Barua, F. Nicholson, T. Ernst and J.W. Doane, 53.4: Novel pulsed drive scheme for improved gray-level uniformity of large-area cholesteric displays, in: SID Symposium Digest of Technical Papers, Wiley Online Library, New Jersey, 2008, pp. 810-813.

[14] T. Xiaoou, Texture information in run-length matrices, IEEE Transactions on Image Processing 7 (1998), 1602-1609.

[15] J.V. Soares, J.J. Leandro, R.M. Cesar, H.F. Jelinek and M.J. Cree, Retinal vessel segmentation using the 2-D Gabor wavelet and supervised classification, IEEE Transactions on Medical Imaging 25 (2006), 1214-1222.

[16] H. Kekre and S.D. Thepade, Image retrieval using color-texture features extracted from walshlet pyramid, ICGST International Journal on Graphics, Vision and Image Processing (GVIP) 10 (2010), 9-18.

[17] M.-M. Cheng, G.-X. Zhang, N.J. Mitra, X. Huang and S.-M. Hu, Global contrast based salient region detection, in: 2011 IEEE Conference on Computer Vision and Pattern Recognition (CVPR), IEEE, 2011, pp. 409-416. 